Tamar Pitch. Catedrática de Filosofía del Derecho del Dipartimento di Giurisprudenza de la Università degli Studi di Perugia, donde enseña también Sociología del Derecho. Se ha interesado mucho por la justicia penal, el control social, el tema criminal, los derechos humanos y el feminismo. Ha sido codirectora de "Studi sulla questione criminale”. Dentro de sus publicaciones más recientes, véanse: Contro il decoro. L'uso politico della pubblica decenza, 2013, Laterza y La società della prevenzione, 2008, Carocci. Contacto: tamar.pitch@unipg.it 


\section{EL TEMA DE LA SEGURIDAD}

Tamar Pitch

Università di Perugia

\section{THE ISSUE OF SECURITY}

DOI: $1017450 / 160105$

Fecha de recepción 10 de noviembre de 2015; fecha de aceptación 11 de enero de 2016. El presente artículo se ha construido en el marco del proyecto de investigación desarrollado en el Dipartimento di Giurisprudenza, Università di Perugia.

\section{Resumen}

Partiendo de la tesis de que el tema de la seguridad compromete a varios discursos, filosóficos, politológicos, sociológicos, cuya fuerza de penetración tiene que ver con los cambios sociales, simbólicos y culturales que atañen al mismo ámbito criminal, este ensayo analiza, antes que todo, la variedad y pluralidad de los significados del concepto "seguridad". Luego, la autora se interesa por los diferentes niveles (global, de la Unión Europea y local nacional) de la seguridad, subrayando la importancia de la perspectiva de género, que sigue siendo muy poco estudiada en la literatura sobre esta materia. Finalmente, muestra cómo el tema de la seguridad incide de forma relevante en el tema criminal, volviendo a trazar sus confines: de la criminalización de nuevos comportamientos a la afirmación de medidas administrativas que tienden a criminalizar determinadas categorías y grupos de personas, que se consideran potencialmente peligrosas.

\section{Palabras clave}

Dispositivos de seguridad, perspectiva de género, ciudadanía, criminalización 


\section{Abstract}

Starting from the assumption that the security issue involves a number of subjects -philosophy, political science, sociology- whose pervasiveness is related to social, symbolic and cultural mutations about criminal issue, this essay analyzes, first of all, the many and various meanings of the security issue. In the second place, the authoress focuses on the different levels (global, within the European Community and national) of security, underlining the importance of genre perspective, so far lacking in the literature about this subject. Finally, she shows how the security issue affects considerably the criminal issue, determining its new borders: from criminalization of new behaviors to the rising of administrative measures that tend to criminalize specific categories and groups of people classified as potentially dangerous.

\section{Key words}

Security devices, gender perspective, citizenship, criminalization

Empezamos este ensayo con la definición del término "seguridad" similar a la afortunada que acuñamos para explicar el término "criminal". El problema es que "seguridad" es un término mucho más polisémico que "criminal", cuyo significado -por mucho que sea tautológico- es claro: lo que la ley considera como tal. Además, desde el punto de vista de los saberes, las ramas que se ocupan del tema criminal son pocas: el derecho, la psiquiatría, la sociología, la criminología. En cambio, hoy día todo el mundo habla de seguridad: filósofos, sociólogos, politólogos, etc. Podríamos limitarnos al ámbito de los significados y de los estudios que más nos interesan y que conocemos mejor (criminología y similares); sin embargo, este mismo ámbito -tanto desde el punto de vista cognoscitivo como de los intereses- está sometido a tensiones y a esfuerzos derivados de la imposición de la "seguridad". Queremos decir que lo de la "seguridad" es un discurso (un conjunto de discursos) cuya actual y difundida relevancia tiene que ver con cambios que atañen al mismo ámbito criminal, y esos cambios poseen, al mismo tiempo, rasgos sociales, simbólicos y culturales. En uno de nuestros últimos ensayos, hemos recorrido toda la "genealogía" de lo que dijimos y escribimos (y creíamos que sabíamos) acerca de la seguridad ${ }^{1}$ Está claro que la

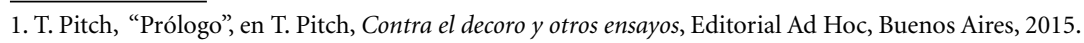


experiencia del comité científico de Città sicure, promovido y estimulado en primer lugar por Massimo Pavarini, fue fundamental en este itinerario, así como fueron fundamentales los estudios y las reflexiones del mismo Massimo Pavarini².

En primer lugar, intentaremos enfocar los temas y los problemas evocados por la "seguridad", además de la variedad de sus significados actuales. En segundo lugar, analizaremos los niveles en los que se inserta el tema de la seguridad y, finalmente, intentaremos evidenciar cómo se relacionan entre sí el tema criminal y el de la seguridad. Está claro que esos tres puntos tienen muchas afinidades.

\section{Algo de literatura}

Un intersante librito de Lucia Zedner ${ }^{3}$ analiza el tema de la seguridad desde muchos puntos de vista e introduce a la perfección la literatura sobre esta materia. Sin embargo, en nuestra opinión, el libro parte de una consideración incorrecta: de hecho, Zedner dice que, hasta hace diez años, la seguridad era casi exclusivamente el ámbito de estudio de los expertos de derecho internacional, de estudiosos de las relaciones internacionales y de la guerra, y que solo recientemente los criminólogos la considerarían central. En realidad, criminólogos, sociólogos de la desviación y del control social, críticos y no críticos empezaron a estudiarla por lo menos desde principios de los años ochenta. Por ejemplo, el proyecto Città sicure fue inaugurado en 1992, tras las experiencias y las investigaciones que llevaban haciéndose desde hacía años en Francia y en el Reino Unido. Los programas ingleses de community safety y los franceses de politique de la ville fueron estimulados y caracterizados por muchas investigaciones sociológicas ${ }^{4} \cdot$ ¿Tal vez

2. M. Pavarini, "Controlling Social Panic: Questions and Answers About Security in Italy at the End of the Millennium", en R. Bergalli, C. Sumner (eds.), Social Control and Political Order. European Perspectives at the End of the Century, Sage, London, 1997, pp. 75-95; M. Pavarini, "Il governo delle città e il bene pubblico della sicurezza", en Minorigiustizia, 2, Franco Angeli, Milano, 1999, pp. 61-69; M. Pavarini, L'amministrazione locale della paura, Carocci, Roma, 2006; M. Pavarini, "Degrado, paura e insicurezza nello spazio urbano”, en Cassazione Penale, 2, Giuffré, Milano, 2009, pp. 389-399; M. Pavarini, "Democrazia e sicurezza", en Ius 17. Studi e materiali di diritto penale, 17, 2, Bologna, 2009, pp. 805-820; M. Pavarini, "La metáfora de la guerra y la democracia de la seguridad”, en Revista de derecho penal, criminología y criminalística, 9, 2012, pp. 3-20; M. Pavarini, M. Donini, Sicurezza e diritto penale, Bononia University Press, Bologna, 2011.

3. L. Zedner, Security. Key Ideas in Criminology Routledge, London, 2009. Véase también G. Procacci, "Le trasformazioni del concetto di sicurezza", en V. Borghi, O. De Leonardis, G. Procacci (eds.), La ragione politica, Vol.II, Liguori, Napoli, 2013, pp. $19-46$. 4. Cfr., por ejemplo, P. Robert (ed.), Les politiques de prévention de la délinquance a l'aune de la recherche. Un bilan International, L'Harmattan, Paris, 1991; A. Crawford, Crime Prevention and Community Safety: Politics, Policies and Practices, Longman, Harlow, 1998; A. Crawford, The Local Governance of Crime. Appeals to Community and Partnerships, Oxford University Press, Oxford, 1999; A. Crawford, "Towards a New Reconfiguration of Powers? Local Orders and the Prospects for Governance through Partnerships", ponencia presentada en la conferencia Normes, déviances, controle social. Nouveaux enjeux, nouvelles approches, París, 14-16 de octubre, 1999; Y. Cartuyvels, P. Mary (eds.), L'Etat face à l'insécurité. Dérives politiques des années 90, Ed. Labor, Bruselles, 1999. 
por un motivo semántico las políticas de community safety no se consideren políticas de security ${ }^{5} \mathrm{Y}$ eso que las primeras tienen las mismas características que se atribuyen a las segundas, antes que todo el hecho de ser dirigidas al futuro, en la base de los principios de prevención y precaución.

Aun antes, en sus seminarios sobre seguridad y territorio de $1978^{6}$, Foucault detectaba en la seguridad el objetivo de la biopolítica. La mayoría de la literatura sobre la seguridad, en Italia y en Francia, parte de o se refiere a Foucault, para quien la seguridad es el objetivo de la biopolítica, un tipo de gobierno que se desarrolla a partir del siglo XIX y que está caracterizado por cuidar al viviente y a las poblaciones. Los objetivos principales de la "seguridad" son eliminar el miedo y la necesidad, buscando prevención y precaución. La salud y el bienestar de las poblaciones, fuente de riqueza y de poder de los estados nacionales, implican el empleo de numerosos dispositivos de gobierno y de control: pasando de la sociedad "disciplinaria" a la sociedad "de la seguridad" -lo que ocurriría alrededor de la segunda mitad del siglo pasado-; control y vigilancia dejarían de ser organizados de forma jerárquica y centralizada (el panóptico) para adquirir rasgos horizontales, anónimos y reticulares. Si la sociedad disciplinaria tenía su "lugar de la seguridad"7 en el cuerpo individual, la sociedad de la seguridad ejercería la seguridad pública sobre la población. Las nuevas tecnologías de vigilancia, además de todos los dispositivos que cada uno de nosotros emplea de forma habitual (móviles, tarjetas de crédito), permiten controlar continuamente los desplazamientos, los gustos, las preferencias, las idiosincrasias, incluso las transgresiones sin que se necesite de un controlador invisible y sin que se interioricen normas sociales o morales. En un ensayo de 1985, retomando algunas sugerencias de Foucault, Stan Cohen ya hablaba de un control que, para la mayoría de la población, aspiraba a observar los comportamientos en lugar de las "mentes". Y una corriente entera -que luego dio lugar a los estudios sobre la vigilancia- analizaba y analiza el funcionamiento y los efectos de las nuevas tecnologías, no solo aquellas que tienen esa determinada finalidad, sino también -y quizás sobre todolas que empleamos por conveniencia o elección 9 .

El contexto social y político en el que se forma la sociedad de la seguridad ha sido descrito de varios modos, que se podrían más o menos resumir en la afirmación de

5. Z. Bauman, In Search of Politics, Stanford University, Press, Stanford (CA), 1999.

6. M. Foucault, Sécurité, territoire, population. Cours au Collège de France (1977-1978), Gallimard/Seiul, Paris, 2004.

7. G. Clavel, La gouvernance de l'insécurité, L'Harmattan, Paris, 2014.

8. S. Cohen, Visions of Social Control, Polity Press, London, 1985.

9. Cfr. D. Lyon, Surveillance Society: Monitoring Everiday Life, Open University Press, London, 2001. Cfr. M. Lianos, M. Douglas, "Dangerization and the End of Deviance. The Institutional Environment", en The British Journal of Criminology, 40, 2, Spring, 2000, pp. 261-78; y N. Rose, “Government and Control”, en Ibid., pp. 321-339. 
un tipo de gobierno neoliberal ${ }^{10}$ respecto de un mundo globalizado, dominado por el capital financiero, en el que se debilitan los Estados nacionales (en realidad no todos) y su capacidad de producir seguridad social ${ }^{11}$ a través de un welfare financiado mediante la tasación progresiva. Los confines cada vez más inestables, la desindustrialización y las siguientes deslocalizaciones, outsourcing, entre otros, con el consiguiente desempleo masivo, precariedad y "flexibilidad" del trabajo, sueldos muy bajos, crisis de los sindicatos y de los partidos tradicionales, desigualdad creciente, la necesidad de convertirse en empresarios de sí mismos sin contar con la ayuda de nadie (el capital humano...) son algunas de las características más destacadas que proporcionan inseguridad y, pues, cada vez mayores exigencias de "seguridad"12. Incluso quien habla de un tránsito del Estado social al Estado penal ${ }^{13}$ hace hincapié en la relación entre crisis del welfare y exigencias de seguridad que se traducirían en "populismo penal ${ }^{14}$ ", con el consiguiente aumento y empeoramiento de la represión penal.

Empleando el término "seguridad" tal y como fue utilizado en Italia, tanto por las políticas como por las retóricas públicas y por gran parte de la literatura, podemos decir que mientras en los "gloriosos treinta" se hablaba sobre todo de "seguridad social" en el sentido de afrontar las eventuales dificultades de la vida gracias a recursos públicos y colectivamente compartidos, a partir de los años ochenta el término seguridad fue empleado sobre todo -aunque no de manera exclusiva- como safety, es decir, como incolumidad personal, salvaguardia de los riesgos de victimización por parte de la delincuencia callejera, del incivismo, etcétera. Esto explica lo que hemos dicho antes.

Sin embargo, la seguridad no solo se exige del Estado y de lo penal. Gran parte de la literatura documenta el gran desarrollo del mercado de la seguridad, con los diferentes aparatos para la protección de sus propias pertenencias, antes que todo de la vivienda; policías privadas y gated communities, y tropas privadas contratadas en todas las guerras actuales. Así que la seguridad se ha convertido (también) en una mercancía, pues en un privilegio cuya adquisición separa aún más a los que pueden permitírsela de los que no, y en algunos casos (por ejemplo, las gated communities) representa una señal de riqueza,

\footnotetext{
10. P. Dardot, C. Laval, La nouvelle raison du monde. Essai sur la société néolibérale, La Découverte, Paris, 2009.

11. Cfr. R. Castel, L'insécurité sociale, Qu'est-ce qu'être protégé?, Seuil, Paris, 2003.

12. Entre otros, véanse Z. Bauman, In Search of Politics; R. Sennett, In The Corrosion of Character: The Personal Consequences of Work in the New Capitalism, W. W. Norton \& Company, New York, 1998; U. Beck, Risikogesellschaft. Auf dem Weg in eine andere Moderne, Suhrkamp Verlag, Berlín, 1986; A. Giddens, The Consequences of Modernity, Polity, London, 1990.

13. Cfr. L. Wacquant, Les Prisons de la misère, Éditions Raisons d'Agir, Paris, 1999. Cfr. A. De Giorgi, Zero Tolleranza. Strategie e pratiche della società di controllo, Derive Approdi, Roma, 2000.

14. J. Pratt, Penal Populism, Routledge, London-New York, 2007.
} 
lo cual no nos induce a considerar la seguridad solo como un "bien público"15. A pesar de no ocuparse claramente de la seguridad, ya al principio de los años ochenta la literatura más específicamente criminológica y mainstream, dejaba de investigar las causas de la criminalidad para defenderse de la misma criminalidad ${ }^{16}$. El hecho de que se pusiera en práctica un enfoque actuarial ${ }^{17}$-que identificara las principales características de las llamadas "poblaciones de riesgo" y/o la búsqueda de medidas penales capaces de neutralizar a los "criminales", la búsqueda de tipos de esterilización del territorio (prevención situacional) y el hecho de dejar de interesarse por el pasado (las causas) para ocuparse del futuro (disminución de los riesgos de victimización)- destaca que el eje de esas criminologías era la seguridad. Simon ${ }^{18}$ ubica en la mitad de los años sesenta el nacimiento en Estados Unidos de un tipo de gobierno through crime, mientras Mary Douglas $^{19}$, hablando del discurso de la politicidad de las elecciones con respecto a los riesgos, se pregunta por lo seguros que deben ser un lugar, una ciudad, una situación, para que se consideren realmente seguros a su vez, subrayando algo bastante desatendido en gran parte de la literatura sobre el tema, es decir, que las llamadas "seguridad objetiva" y la "seguridad subjetiva" no se pueden distinguir y que la "seguridad" siempre atañe a una comparación entre estados y situaciones.

Además, la mayoría de la literatura interroga acerca del llamado "paradigma victimario". Queda confirmada la centralidad de la figura de la víctima en las políticas y en las retóricas sobre la seguridad ${ }^{20}$. Sin embargo, la figura de la víctima o incluso el paradigma victimario ${ }^{21}$ están por doquier, tanto en las retóricas públicas como en los estudios e investigaciones. Para la literatura a la que nos referimos, esta ubicuidad (y centralidad) implica que la víctima es la otra cara del sujeto neoliberal22, es decir que a su vez, muestra la importancia de la seguridad en el tipo de gobierno neoliberal. En nombre de las víctimas, actuales o potenciales (incluidos nosotros), no solo se

15. Cfr. M. Pavarini, "Il governo delle città e il bene pubblico della sicurezza", Zedner, citando a Pavarini, sostiene lo mismo, véase a L. Zedner, Security.

16. Cfr. D. Garland, The Culture of Control: Crime and Social Order in Contemporary Society, Oxford University Press, Oxford, 2001.

17. Cfr. M. Feeley, J. Simon, Actuarial Justice: The Emerging New Criminal Law, en D. Nelken (ed.), The Futures of Criminology, Sage, London, 1994; P. O’Malley, “Risk, Power and Crime Prevention”, in Economy and Society, 21, 3, 1992, pp. 252-275. 18. J. Simon, Governing through Crime. How the War on Crime Transformed American Democracy and Created a Culture of Fear, Oxford University Press, New York, 2007.

19. M. Douglas, Risk Acceptability According to the Social Sciences, Sage, London, 1986; Risk and Blame: Essays in Cultural Theory, Routledge, London, 1992.

20. En muchas criminologías, incluídas las “de izquierda”, véase también D. Garland, The Culture of Control.

21. Cfr. D. Giglioli, Critica della vittima, Nottetempo, Roma, 2014; G. De Luna, La repubblica del dolore, Feltrinelli, Milano, 2011 .

22. Cfr. W. Brown, “American Nightmare. Neoliberalism, Neoconservatism and de-democratization", en Political Theory, December, 34, 6, 2006, Sage, pp. 690-714. 
ponen en práctica o justifican políticas represivas y preventivas por parte de los gobiernos locales y nacionales, sino que también se lleva a cabo una organización colectiva, una movilización, se adquiere voz. La categoría de la llamada "ciudadanía activa” no es pequeña y está constituida precisamente por grupos de "víctimas" o potenciales víctimas (llevando a "comunidades de cómplices" 23 o a comunidades excluyentes ${ }^{24}$ ), un fenómeno antiguo, pero que hoy está mucho más difundido que hace veinte años, por haber disminuido aquel tipo de agregación y de movilización que no se fundamentaban en el miedo (sindicados, partidos de masas).

Por lo tanto, destacamos el protagonismo de la "víctima” tanto en el discurso científico como en el político. Los motivos son muchos, partiendo de los análisis filosófico-políticos como el de Wendy Brown ${ }^{25}$ que, en la figura de la "víctima", vislumbra el estatuto de la víctima que brota del cruce entre neoliberalismo y neoconservadurismo, y llega a los sociológicos y criminológicos. Por ejemplo, Jonathan Simon ${ }^{26}$ pone de manifiesto la progresiva afirmación, en Estados Unidos, de lo que él llama "gobierno mediante la criminalidad", es decir, una modalidad de gobierno inspirada en la lógica de lo penal y basada en el miedo, que se impone en muchos ámbitos sociales: escolar, ciudadano, empresarial y en las modalidades de distribución del welfare. Hay muchas conexiones entre la hegemonía neoliberal y neoconservadora (véase Brown por lo que a las diferencias y complementariedades de esas racionalidades políticas se refiere) y los resultados trazados por Simon. Al principio de los años ochenta, Thatcher en el Reino Unido y Reagan en Estados Unidos discutieron sobre el welfare y sus culturas: Thatcher sostenía que la sociedad no existía, que solo existían los individuos, mientras que Reagan negaba la existencia de la criminalidad como problema social y con causas sociales: solo se enfrentan los buenos y los malos y a los malos hay que combatirlos. Así que si -como decía Thatcher- la sociedad no existe, ni siquiera existen los llamados problemas sociales y la misma "criminalidad", sino solo -como decía Reagan- personas "malas" que se deben combatir y reprimir. Atacando el welfare, pasamos de los criminales y de las causas que los inducen a delinquir, a las víctimas y a las maneras de defenderlas.

Ya en $1989^{27}$ parecía que responsabilidad y victimización tuvieran muchas relaciones por la lógica de lo penal, donde responsabilidad solo significa causalidad de la acción

23. S . Bauman, In Search of Politics.

24. M. Feeley, J. Simon, Actuarial Justice.

25. W. Brown, "American Nightmare", pp. 690-714.

26. J. Simon, "Theaching Criminal Law in an Era of Governing through Crime", en Law Journal, 48, Saint Louis University, 2004, pp. 1313-1335.

27. Cfr. T. Pitch, Responsabilità limitate, Feltrinelli, Milano, 1989. 
y pleno conocimiento, y víctima es quien sufrió un daño por parte del responsable. Ambos pierden cualquier otra característica social, cultural e incluso de "género" y también desaparece el contexto donde se origina la acción. Hay muchas similitudes con el sujeto neoliberal. De hecho, este es un individuo cuya libertad estriba en la total asunción de responsabilidades respecto de elecciones que derivarían de una racionalidad utilitarista, y también en su capacidad de pagar solo los eventuales precios. La libertad de este sujeto se acepta de forma apriorística y se considera que no depende de ningún contexto social, económico, institucional y político. Individualización y privatización de elecciones y costes se alejan claramente de la racionalidad política típica de los estados de welfare, donde ambos de alguna manera eran compartidos por la colectividad por medio de medidas de protección y de compensación de los riesgos, además de redistribución de la riqueza, y cuyas elecciones y costes dependían del contexto en el que ellos se daban. El rasgo de victimario de este sujeto reside en la coeva afirmación de la seguridad por parte del discurso político dominante, que legitima la actividad del Gobierno. Esta afirmación es totalmente clara y explícita por lo que atañe al tema criminal. La división entre ofensores y víctimas potenciales (todo el mundo) no solo sirve para separar a la gente buena de la mala (y, pues, para construir las nuevas clases peligrosas, caracterizadas por quien no puede correr peligros en el mercado del trabajo porque no puede pagar las eventuales consecuencias negativas), sino también para lograr el consenso de las clases medias emprobrecidas por la crisis económica y más preocupadas por el cambio del mercado del trabajo - cada vez más precario y menos disponible- y por los recortes al welfare procedentes de las decisiones neoliberales que por la criminalidad. Más en general, estamos frente a una privatización y moralización del discurso público ${ }^{28}$, en el que la acción del Gobierno se justifica como defensa de las "víctimas". La desviación de la atención de los criminales a las víctimas implica renunciar a proyectos de reforma social y/o de rehabilitación personal por ser inútiles y costosos -incluso contraproducentes por ser desresponsabilizantes y "laxistas" - y razonar en la base de una óptica que une ${ }^{29}$ el utilitarismo neoliberal y el discurso moralizante neoconservador.

En un libro publicado en $1996^{30}$, Garapon también registraba un cambio (en Francia) de la justificación de la pena, que ya no se consideraba una manera de tratar al criminal, de restablecer el orden o de volver a imponer la ley, sino en cambio, una medida para satisfacer a la víctima. De ahí que la pena fuera una compensación psicológica.

28. W. Brown, "American Nightmare".

29. Ibid.

30. A. Garapon, D. Salas, La République pénalisée, Hachette, Paris, 1996. 
Garapon también deducía, en consecuencia, que ahora el Estado justificaba su acción poniéndose al servicio de los sufrimientos privados. Así que el nacimiento de una "sociedad de víctimas" incluso implicaba una creciente privatización del Gobierno y una especie de homologación de los escenarios social y penal con evidentes rasgos "moralizantes"; de hecho, neutralizar a los malos no solo les era útil a los buenos para protegerlos de las acciones de los malos, sino también para indemnizar por el padecimiento sufrido a los afectados por esas acciones. Así que la pena se refiere a la venganza privada y a aquella de la sociedad. La justificación retributiva -al mal se reacciona con otro malse relaciona con la retribución privada, a menudo muy claramente, como cuando no se infligen penas alternativas porque no lo quieren aquellas víctimas específicas. De alguna manera la introducción de la mediación penal responde a una lógica semejante ${ }^{31}$.

A partir de finales de los años ochenta, hay una convergencia de intereses entre estudios que se interrogan acerca del tema de la seguridad en el ámbito local y nacional y estudios que hasta aquel entonces se habían interesado por la llamada seguridad externa, es decir, la protección de los confines. La finalización de la Guerra Fría con el derrumbe del muro de Berlín implicó una reconversión de los tradicionales security studies, hasta aquel entonces dedicados al estudio de la seguridad externa, es decir, a la seguridad del Estado y, al mismo tiempo, un cambio del significado mismo de seguridad. El fin de un territorialized enemy, tal y como lo llama Bigo $^{32}$, no solo llevó a reconsiderar la seguridad y a una larga serie de debates sobre el estatuto del cambio de paradigma introducido por la llamada Escuela de Copenhague ${ }^{33}$, sino también a una ampliación de los ámbitos y de los fenómenos examinados en ese paradigma.

En el debate y en los estudios sobre la seguridad y la securitización no siempre se entiende si las novedades en la literatura dependen de los cambios en el uso político de la categoría de seguridad, o bien si tienen que ver con los cambios políticos (la desaparición, en Europa y en Estados Unidos, de un “enemigo territorializado", una desaparición momentánea por lo que parece) y la consiguiente necesidad de expertos del sector de renovar sus saberes. Además, no está muy claro si los cambios de los que hablan los nuevos estudios críticos sobre la seguridad atañen al ámbito de los discursos políticos y de aquellos de los expertos, o bien a los cambios concretos, es decir, relativos a las

31. O. De Leonardis, T. Pitch, “Un mundo horizontal. Las rétoricas y las prácticas contemporáneas de la mediación”, en M. Sozzo (ed.), Por una sociología crítica del control social. Ensayos en honor de Juan S. Pegoraro, Editores Del Puerto, Buenos Aires, 2011.

32. Cfr. D. Bigo, "Protection: Security, Territory and Population", en J. Huysmans, J.A. Dobson, R. Prokhovnik (eds.), The Politics of Protection. Sites of Insecurity and Political Agency, Routledge, London, 2006, pp. 84-100.

33. B. Buzan, O. Waever, J. de Wilde, Security: A New Framework forAnalysis, Lynne Rienner, Boulder-London, 1998. 
nuevas condiciones geopolíticas. Lo cierto es que, a partir de los años noventa, los estudios sobre la seguridad, en otras palabras, sobre los procesos de securitización, se multiplican y a veces se cruzan, ignorando los trabajos anteriores de los estudios sociológicos y criminológicos. Lo que consideramos valioso de esta literatura es que se interesa por los procesos de securitización. La Escuela de Copenhague protagoniza este cambio de itinerario. Tras definir la "seguridad" como "estar libre de amenazas a la supervivencia”, esta escuela opina que la securitización es un acto lingüístico por parte de quien detiene el poder, que produce un determinado fenómeno, acontecimiento y proceso como "amenaza grave a la incolumidad, a la supervivencia" de alguien o algo, desplazando el fenómeno, acontecimiento o proceso del ámbito de la política ordinaria al de la emergencia. Se trata de un desplazamiento que, frente a la emergencia, lleva a decisiones que se legitiman en la base de la necesidad urgente de hacer frente al peligro de la amenaza y que, pues, no forman parte de la normal dinámica política. Esta escuela, así como muchos otros autores -tanto los que aceptan el paradigma que ella propone como los que lo critican parcial o totalmente- destacan que muchos Gobiernos, y en general las élites políticas, recurren cada vez más a la "securitización" que, en las sociedades democráticas, desplazaría de forma significativa el poder en las manos del ejecutivo, en detrimento de la discusión y de la decisión parlamentarias. Esto también llevaría a un aumento de los ámbitos, de los fenómenos, de los temas y de los procesos relacionados con ella.

Y si el tema más estudiado hasta ahora es el cambio de la inmigración (en Europa y en EE. UU.) de términos económicos o humanitarios a términos de seguridad ${ }^{34}$, muchos estudios sobre la securitización se ocupan de otros temas, como el medio ambiente y la humanidad. De ahí que recurramos a numerosos discursos y políticas sobre materias diferentes que remiten todas al apelativo "seguridad". Esta ampliación de los ámbitos en los que se invoca la protección implica consecuencias tanto en lo referente a la manera de entenderlos y construirlos como en su análisis (pénsemos, por ejemplo, en la significativa distorsión del tema, de todas formas controvertido, del "desarrollo"). A su vez, el significado de seguridad se ensancha hasta perder valor; sin embargo, su empleo desproporcionado pone de manifiesto que hoy la "seguridad" se ha convertido en tema fundamental en los discursos y en las políticas. La seguridad parece haberse convertido en la modalidad discursiva prevaleciente para analizar los problemas sociales e indicar su itinerario final. La Comisión sobre la Seguridad Humana de la ONU

34. Véase, por ejemplo, J. Huysmans, The Politics of Insecurity. Fear, Migration and Asylum in the European Union, Routledge, London, 2006. 
(2000) es la prueba de cómo también los derechos humanos son englobados en la "seguridad", que mejor que ellos sabría detectar lo más necesario y deseable a nivel político. La seguridad ya no es la condición mínima necesaria para la democracia, la libertad, el desarrollo humano, sino la finalidad que la política debe lograr. De instrumento se convierte en objetivo, de ahí que nos alejemos bastante también de la seguridad de Hobbes $^{35}$, según el cual ella era una condición necesaria y no el objetivo último de la acción colectiva e individual ${ }^{36}$.

Sin embargo, no todos los discursos sobre la seguridad son securitarios, en el sentido empleado y teorizado por los critical security studies. Ciertamente no lo sea la "seguridad humana" exigida por la ONU, a pesar de que -como lo subraya Foessel ${ }^{37}$ - el paradigma de la seguridad humana aumenta el riesgo de confusión entre el deseo legítimo de estabilidad existencial y la finalidad de la relación política. Tal vez también en esa ocasión se trate de un problema de términos pero, por ejemplo en Italia, la desviación del significado de prevalencia social de seguridad al significado actual señala la crisis del Estado social y sus consecuencias, lo que destaca no solo -como en los critical security studiesla progresiva falta de distinción entre interior y exterior y la progresiva confusión entre enemigos y criminales, sino también que estos procesos tienen que ver con cambios procedentes no solo de la terminación de la Guerra Fría, sino también de la gradual hegemonía de políticas neoliberales y de una general racionalidad neoliberal.

Lo que destacamos es la utilidad del enfoque de la Escuela de Copenhague para el análisis de lo que la securitización conlleva: si no conlleva despoliticización, de seguro conlleve la desviación de los temas respecto de los cuales el debate político ordinario la aplica al registro de las urgencias y, pues, de la decisión por parte del ejecutivo. Pero también los estudios de los critical security studies contribuyen a destacar la casi inevitable escalation producida por los discursos y por los procesos de securitización, ya registrada por los sociólogos ${ }^{38}$ y por los criminólogos. Además, la entrada de un tema en el registro discursivo de la seguridad ayuda a solucionarlo; es la solución que construye el problema y no al contrario. Y puesto que el discurso securitario es un discurso de la amenaza, de la urgencia y de la emergencia, este tenderá a ocultar o a englobar en su interior políticas incoherentes con la amenaza, con la urgencia y la emergencia, lo que ha ocurrido -lo explicaremos mejor más adelante- en la mayoría de los casos en el ámbito

35. M. Foessel, Etat de vigilance. Critique de la banalité sécuritaire, Le Bord de l'eau, Lormont, 2010.

36. Respecto de la relación entre seguridad y derechos humanos, cfr. B. J. Goold, L. Lazarus (eds.), Security and Human Rights, Hart Publishing, Oxford/Portland (OR), 2007.

37. M. Foessel, Etat de vigilance, p. 103.

38. Cfr., por ejemplo, R. Castel, L'insécurité sociale, Qu'est-ce qu'être protégé? 
de las políticas locales de seguridad, incluso cuando estas tenían buenas intenciones y programas que intentaban integrar políticas de esterilización del territorio con políticas inclusivas. Entonces, esto ha ocurrido por todas las razones que hasta aquí hemos subrayado (tendencia a la escalation, desviación en el registro de la urgencia, conexión solución-problema): la referencia a la seguridad -en el sentido de safety-acaba dejando de lado las políticas sociales, haciendo prevalecer aquellas de tipo represivo. Además, la desviación de la inmigración, por ejemplo, de tema económico o humanitario al de seguridad lleva a políticas inmigratorias cada vez más restrictivas y penalizantes, que empujan a los migrantes hacia los territorios de la ilegalidad ${ }^{39}$

En el amplio panorama de la literatura de la seguridad, cabe referir a dos filósofos ya citados, Wendy Brown y Michel Foessel. De la primera, además de la obra sobre neoliberalismo y neoconservadurismo en Estados Unidos -que citamos antes con respecto a la figura de la víctima ${ }^{40}$, es muy importante el ensayo sobre las nuevas barreras ${ }^{41}$. Los nuevos muros que se han construido por doquier (Brown recuerda el muro entre Estados Unidos y México; Israel y Palestina; Ceuta y Melilla; la India y Pakistán, entre otros, y también recuerda el muro de Via Anelli en Padua, además de las gated communities) después de veinte años de la caída del Muro de Berlín son barreras cuyo alcance es simbólico, aunque sus consecuencias son reales y a menudo trágicas. En un mundo en apariencia uniforme por los continuos flujos de mercancías, dinero, comunicaciones y también personas, se construyen muros en cada esquina ${ }^{42}$, y se trata de muros que deberían alejar las amenazas y los peligros procedentes del exterior (migrantes, terroristas, extranjeros, criminales, traficantes de droga, solicitantes de asilo, pobres en general), es decir, reconstruir, o incluso crear un Estado-nación, o una autoridad, pues unos muros de protección, nuevos homines muniti, porque estos muros, a diferencia de los de las cárceles y de los varios campos, no se construyen para "incluir" sino para "excluir", como las paredes de las antiguas ciudades fortificadas. Y, como siempre ha ocurrido, producen efectos no solo en el exterior, sino también y sobre todo en el interior, por lo que contribuyen a construir subjetividades pasivas, paranoicas, xenófobas, en consecuencia, homines muniti. Podríamos preguntarnos cuál es el confín entre incluir y excluir, es decir, cuánto o cuándo estos nuevos muros acaban pareciéndose a los de las cárceles.

\footnotetext{
39. Pitch analizó el ejemplo de los gitanos, a menudo obligados al nomadismo por políticas que los "construyen" precisamente como "nómadas" y que, pues, no pueden integrarse en el tejido urbano, véase T. Pitch, Contro il decoro. L'uso politico della pubblica decenza, Laterza, Roma-Bari, 2013.

40. Cfr. W. Brown, "American Nightmare".

41. Véase M. Foessel, Etat de vigilance.

42. M. Teheranian, J. Camilleri y K. Malhotra, Reimagining the Future: Toward Democratic Governance, Bundoora, Australia: Department of Politics, La Trobe University, 2000.
} 
En este punto podemos recordar, en el caso de los muros, lo que dijimos con respecto a las medidas que llamamos políticas para la seguridad a nivel local y nacional: ellos sirven simbólicamente para separar a los buenos de los malos, mejor dicho, para considerar a los incluidos como buenos y a todos los demás como malos, y para intentar construir una relación entre los buenos basada en el miedo.

De hecho, esta interpretación nos explica incluso el aumento del mercado privado de la seguridad, que no solo se añade, sino que también interactúa ${ }^{43}$ con los dispositivos securitarios públicos: los homines muniti; es decir, las subjetividades construidas sobre la base de la desconfianza y el miedo al otro están dispuestas, en otras palabras, son invitadas a defenderse también a solas si tienen los recursos económicos para hacerlo, tal y como sucede en el ensayo sobre las relaciones entre neoliberalismo y neoconservadurismo en Estados Unidos. Brown ${ }^{44}$ muestra la aparentemente contradictoria pero fructuosa alianza entre modalidades de control interpersonales, basadas en la "libertad" individual y modalidades que, en cambio, se fundamentan en las "mentes" ${ }^{45}$, por medio de retóricas “moralizantes”. Y eso que el hecho de hacer a solas, de convertirse en empresarios de sí mismos y de asumir toda la responsabilidad de sus acciones y de las relativas consecuencias es de por sí un imperativo "moral" ${ }^{6}$.

Recordando ese ensayo de Brown, Foessel ${ }^{47}$ se interroga acerca de lo que él llama banalidad securitaria, relacionando el aumento de la demanda de seguridad con el desengaño tanto respecto de la política como del derecho, dudando de que el significado actual de seguridad pueda derivar de Hobbes: para Foessel, la demanda de seguridad se presenta como una modalidad individual, moral y prepolítica, como si el modelo securitario puesto en práctica en esas últimas décadas invalidara la tesis según la cual solo un poder público puede solucionar el problema del miedo ${ }^{48}$. Además de su "naturalización", Foessel subraya la "seguridad" como finalidad, individual y colecti$\mathrm{va}^{49}$. En realidad, diríamos que solo se trata de una naturalización en el sentido de que, por medio de la securitización, las amenazas se presentan como "objetivas", en lugar de resultado de elecciones políticas ${ }^{50}$; de lo contrario, todos los “peligros" y, lo más importante, todo todos los acontecimientos perjudiciales tienden a atribuirse a la acción o a la

\footnotetext{
43. Por medio de numerosos instrumentos, véase el resumen y la bibliografía presentada en L. Zedner, Security. 44. W. Brown, "American Nightmare".

45. S. Cohen, Visions of Social Control.

46. Cfr. M. Douglas, Risk Acceptability According to the Social Sciences; Ead., Risk and Blame; T. Pitch, "I rischi della sicurezza urbana”, en Parolechiave, 22-23-24, 2000, pp. 71-97; Ead., La società della prevenzione, Carocci, Roma 2006.

47. M. Foessel, Etat de vigilance.

48. Ibid.,p. 18.

49. Ibid., p. 104.

50. Cfr. M. Douglas, Risk and Blame; T. Pitch, La società della prevenzione.
} 
omisión de alguien ${ }^{51}$. Ya dijimos precisamente que esto fundamenta la gran difusión del imperativo de la prevención, tanto individual como privada, tanto pública como colectiva, cuyo imperativo, a su vez, se da a conocer al modelo securitario.

Este breve excursus sobre una parte de la literatura sobre la seguridad producida en ámbitos disciplinarios diferentes permite descifrar convergencias y divergencias a la hora de analizar el fenómeno; tal y como ya ponía de manifiesto antes, las convergencias atañen al ámbito general de su relación con la imposición de una racionalidad política neoliberal; probablemente las divergencias tengan que ver con aspectos diferentes del fenómeno mismo. Por ejemplo, quien se ocupa de políticas securitarias en los diferentes ámbitos en que se manifiesten no solo tiende a descuidar la existencia de un gran mercado privado de la seguridad, sino también la relación entre público y privado ${ }^{52}$ que, en cambio, es fundamental para entender cómo cambia el tema criminal (del que hablaremos luego). Quien subraya que la gubernamentalidad neoliberal actúa por medio de la libertad individual y modalidades de control impersonales desatiende tanto los aspectos éticos y morales de esa misma gubernamentalidad como sus aspectos claramente coercitivos y represivos.

\section{Los niveles}

La seguridad en ámbito local y nacional es analizada sobre todo por sociólogos, criminólogos y antropólogos. Las investigaciones sobre el ámbito local son muy numerosas $\mathrm{y}$, como hemos mencionado antes, comienzan a partir de los años ochenta ${ }^{53}$. También en Italia hay muchos estudios sobre la victimización, el "miedo a la criminalidad", las políticas de seguridad urbana ${ }^{54}$, y en este ámbito, el proyecto Città sicure de seguro fue

\footnotetext{
51. Ibid.

52. Véase L. Zedner, Security y T. Pitch, La società della prevenzione, Carocci, Roma, 2006.

53. Una obra muy importante y poco citada por los criminólogos es el estudio de E. S. Merry, Urban Danger. Life in a Neighborhood of Strangers, Temple University Press, Philadelphia, 1981.

54. Véanse, por ejemplo, G. Amendola (ed.), Paure in città. Strategie ed illusioni delle politiche per la sicurezza urbana, Liguori, Napoli, 2003. Véanse Id., (ed.), Il governo della città sicura, Liguori, Napoli, 2003; F. Carrer, Sicurezza in città e qualità della vita, Roma, Editrice Libertà, 2000; A. Ceretti, R. Cornelli, Oltre la paura. Cinque riflessioni su criminalità, società e politica, Feltrinelli, Milano, 2013; M. Barbagli, L. Sabbadini, La sicurezza dei cittadini, il Mulino, Bologna, 1999; G. Mosconi, Criminalità, sicurezza e opinione pubblica in Veneto, Cleup, Padova, 2000; Id., "La sicurezza dell'insicurezza. Retoriche e torsioni della legislazione italiana”, en Studi sulla questione criminale, 2, mayo-agosto 2010, pp. 75-99; A. Dal Lago, E. Quadrelli, La città e le ombre. Crimini, criminali, cittadini, Feltrinelli, Milano, 2003; M. Pavarini (ed.), L'amministrazione locale della paura, Carocci, Roma, 2006; T. Pitch, C. Ventimiglia, Che genere di sicurezza. Donne e uomini in città, Franco Angeli, Milano, 2001; R. Selmini, "Le politiche di sicurezza in Italia. Origini, sviluppo e prospettive" en M. Barbagli (ed.), Rapporto sulla criminalità in Italia, il Mulino, Bologna, 2003, pp. 611-648; R. Selmini, "Paura, rischio e insicurezza tra domanda sociale e culture politiche: Torino, Bologna, Napoli”, en G. Amendola, Il governo della città sicura; Ead. (ed.), La sicurezza urbana, il Mulino, Bologna, 2004.
} 
pionero (véase los Quaderni de Città sicure). Vamos a ver cuáles son, muy brevemente, los resultados más destacados de este gran trabajo. A pesar de su parcial diferencia entre ellas y a pesar de ser muy buenas, las políticas de seguridad implementadas a nivel local a menudo son englobadas en la "securitización". Así que se impone la referencia a la seguridad como incolumidad de los ciudadanos respecto de las amenazas de criminalidad callejera, del incivismo, de las ilegalidades difundidas y sugiere medidas dirigidas también a la llamada "prevención social" (servicios sociales específicos, asistencia a las víctimas, saneamiento del territorio, aumento de la sociabilidad, etc.), total que los gobiernos locales (nos referimos a Italia) aspiran explícitamente no tanto al aumento de la seguridad, sino a la disminución de la percepción de inseguridad. La mayoría de los resultados de estos gobiernos ha sido importante sobre todo desde el punto de vista simbólico. Pensemos en la llamada temporada de las ordenanzas ${ }^{55}$, que aún no ha acabado (véase, por ejemplo, la reciente ordenanza del alcalde de Padua Bitonci). Todos o casi todos los gobiernos locales, de todo tinte político, han dictado ordenanzas similares (incluso a veces copiadas las unas de las otras), cuyo objetivo es la esterilización del territorio, eliminar lo que se considera perjudicial, contaminante, enemigo, amenazador. Los blancos de estas ordenanzas son fáciles de detectar: antes que todo gitanos, prostitutas, mendigos, grafiteros, vendedores ambulantes, tiendas de comida "étnica" ${ }_{56}$.

En Francia, la situación no es muy diferente ${ }^{57}$. En cambio, Reino Unido fue uno de los primeros en equiparse con cámaras de circuito cerrado por doquier, algo que luego se difundió también en Italia, incluso en los pueblos más pequeños. Cabe añadir que ni las ordenanzas, ni las cámaras han logrado el objetivo que se habían propuesto. Las ordenanzas casi no se ponen en práctica, aunque hayan llevado simbólicamente a una división entre buenos y malos. Las cámaras se rompen enseguida y se quedan así, porque arreglarlas o sustituirlas cuesta demasiado. Su utilidad todavía no ha sido demostrada: tal vez sirvan para detectar a los transgresores, después de haber cometido el crimen. Sin embargo, lo fundamental es que la discusión sobre la seguridad ya se ha convertido -en todos los ámbitos- en un recurso político importante: se emplea para cosechar éxitos, para lograr fondos y para promover el disenso, con consecuencias incluso graves como las incursiones contra los llamados campos nómades y, de manera más reciente, contra los centros de acogida de los migrantes y los solicitantes de asilo.

55. M. Giovannetti (ed.), Per una città sicura, Roma, Cittalia, 2012 y Id., "Le ordinanze dei sindaci sulla sicurezza urbana”, en ibid., pp. 25-60.

56. T. Pitch, Contro il decoro.

57. V. Gautron, Les politiques publiques de lutte contre la délinquance, Université de Nantes, 2006. 
Así que si muchos proyectos y experimentos locales intentaban (e intentan) ocuparse tanto de las preocupaciones de los ciudadanos acerca de la habitabilidad y del empleo de los espacios urbanos como de las exigencias de que se respeten los derechos de todos y de todas las que emplean estos mismos espacios (lo cual no es nada fácil, por lo menos en Italia, cuando los recursos de los que disponen las autoridades locales disminuyen cada vez más) y si hay buenos instrumentos que se pueden emplear tanto en Italia como en otras partes (por ejemplo, Francia y Reino Unido), más se ha aspirado a esterilizar el territorio urbano y a estigmatizar una parte de la población urbana que a reconstruir relaciones sociales y producir confianza generalizada ${ }^{58}$. Era de esperarse.

Los migrantes y los solicitantes de asilo - no solo en Italia- se han convertido en el objetivo principal de las políticas de seguridad a nivel nacional. La criminalización de la inmigración en los países europeos y en EE. UU. se ha difundido tanto como para haber creado un nuevo fenómeno, llamado crimmigration ${ }^{59}$. Los estudios sobre este tema son muy numerosos. En el ámbito de la Unión Europea, el cambio de la inmigración desde terceros países por razones económicas o humanitarias a relacionadas con la seguridad ha sido analizado por los critical security studies. Por lo que a los contextos nacionales se refiere, es suficiente analizar los detenidos para entender los efectos de esas políticas, por ser los extranjeros superrepresentados (otro tema es la prisión administrativa, es decir, los diferentes campos, centros de acogida e identificación, diseminados en toda Europa). Los llamados paquetes de seguridad promulgados en Italia en los últimos años atañen no solo a los migrantes, sino también a un conjunto de fenómenos y figuras (piénsese en el "feminicidio") caracterizados por ser "emergencia”. En Italia, pero también en Francia, una de estas "emergencias" han sido los gitanos.

Si, por una parte, sobre todo criminólogos y sociólogos han estudiado la seguridad en el ámbito local y nacional, por otra parte, las políticas de seguridad de la UE han sido analizadas en especial por los critical security studies (la ya citada Escuela de Copenhague), sobre todo con relación a la inmigración y a la solicitud de asilo de personas procedentes de terceros países, aunque esas políticas -como veremos- también atañen a los ciudadanos europeos.

La "securitización" de la inmigración en las políticas europeas es algo anterior tanto al 11 de septiembre como al fin de la Guerra Fría. Como subraya Huysmans ${ }^{60}$, a partir de la mitad de los años ochenta, en el proceso de integración europeo se desarrolla un

58. T. Pitch, "I rischi della sicurezza urbana".

59. J. Stumpf, “The Crimmigration Crisis", en American University Law Review, 56, 2, Washington College of Law, Washington, 2006, pp. 1-55.

60. J. Huysmans, The Politics of Insecurity. 
ámbito de seguridad interna que pone en relación temas de control de los confines, terrorismo, drogas, delincuencia organizada y solicitud de asilo, sobre todo a partir de los años noventa. Puede parecer paradójico que las medidas de seguridad se conviertan en relevantes para la UE justo cuando un "enemigo territorializado ${ }^{61}$ " desaparece y se construye un espacio de libertad de movimiento para los ciudadanos europeos con los acuerdos de Schengen (1985). Esta paradoja se interpreta de dos formas: por un lado, en especial para los que hacen hincapié en la acción de las agencias de seguridad ${ }^{62}$, el proceso de securitización puede ser imputado, por lo menos por una parte, precisamente a la necesidad de los burócratas y de los expertos de seguridad de volver a orientar y definir sus tareas en un contexto que ha cambiado; por otra parte, este mismo proceso se puede considerar coherente con y útil para una racionalidad política neoliberal, mientras la seguridad está directamente relacionada con el impulso a la movilidad de poblaciones, mercancías y servicios ${ }^{63}$. De ahí que una mayor libertad para los ciudadanos europeos y mayores restricciones para la movilidad de ciudadanos de terceros países impliquen una "noción liberal de seguridad", según la cual se logra un mayor control gracias a medidas administrativas en lugar de prohibiciones legales. De hecho, como evidencia van Munster, mientras la movilidad de los ciudadanos europeos aumenta, la movilidad de los migrantes intenta ser inmovilizada por considerarse un peligro, y si logran moverse, su movimiento es controlado por tecnologías de gestión del riesgo ${ }^{64}$. A través de la construcción de "poblaciones potencialmente peligrosas", riesgo y gestión del riesgo fundamentan las políticas de seguridad de la UE y los cambios de las políticas criminales analizados por sociólogos y criminólogos en las últimas tres décadas.

Podríamos decir que los acuerdos de Schengen han facilitado la movilidad de los ciudadanos europeos y, al mismo tiempo, han dificultado a los ciudadados de terceros países la posibilidad de entrar y moverse en este espacio. En 1990, la Convención para la aplicación de los acuerdos de Schengen ya pone en relación la inmigración con el terrorismo y la criminalidad transnacional, incluyendo las normas relativas a la inmigración en un contexto institucional dedicado a la defensa de la seguridad interna. Mientras Schengen 1 había sido negociado y escrito por funcionarios expertos de transporte y

61. D. Bigo, "Security, Territory and Population".

62. Cfr., por ejemplo, D. Bigo, "From Foreigners to 'Abnormal Aliens'. How the Faces of the Enemy Have Changed", en E. Guild, J. van Selm (eds), International Migration and Security: Opportunities and Challenges, Routledge, London, 2005, pp. 64-81.

63. R. van Munster, Securitizing Immigration. The Politics of Risk in the European Union, Palgrave Macmillan, New York, 2009 p. 98.

64. Ibid., p. 98; Lianos y Douglas han documentado el mismo proceso a nivel nacional, cfr. M. Lianos, M. Douglas, "Dangerization and the end of deviance. The institutional environment", pp. 261 -78. 
de asuntos exteriores, Schengen 2 lo han escrito profesionales de la seguridad interna. En los acuerdos de 1985 el tema de la seguridad no era fundamental, luego lo llegó a ser. Y después del 11 de septiembre, la conexión entre la inmigración y el riesgo del terrorismo es concreta (cfr. los arts. 16 y 17 del European Council Common Position on Combating Terrorism, diciembre de 2001). Por lo tanto, los migrantes y los solicitantes de asilo pasan de ser un problema económico o humanitario a un problema de seguridad (también en el ámbito nacional nos enfrentamos con similar desviación).

Está claro que los migrantes y los solicitantes de asilo son la preocupación más candente de las políticas de seguridad de la UE, pero no son la única. Los acuerdos de Schengen y los documentos siguientes implican restricciones legitimadas por problemas de seguridad también para los ciudadanos europeos. Estas restricciones pueden ser aplicadas por los Estados contrayentes cuando se tiene la sospecha de que su seguridad corre peligro, pero ellos mismos deciden cómo y cuándo aplicarlas, así que estas decisiones son totalmente discrecionales. Se pueden bloquear en las fronteras a los que se consideren "perturbadores potenciales", pero la molestia potencial se deja a la discrecionalidad de cada país en la base de la "sospecha" y de cálculos de riesgo, es decir, superando cualquier evidente estándar legal ${ }^{65}$ (un ejemplo italiano fue Génova 2001). Por lo tanto, políticas creadas para combatir el terrorismo y la inmigración ilegal también se aplican a los ciudadanos europeos, considerados "sospechosos". En este caso, no solo se niega la libertad de movimento, sino también se menoscaban otros derechos fundamentales, como la libertad de expresión, porque se define "sospechoso" al sujeto en la base de sus propias ideas políticas. Así que aproximaciones, lenguajes y técnicas desarrolladas en nombre de la seguridad contra el terrorismo, la delincuencia organizada y la inmigración acaban atañendo también a los ciudadanos europeos.

Hay otra población de ciudadanos europeos considerada potencialmente peligrosa, cuyos derechos pueden ser limitados en nombre de la seguridad: hablamos de los llamados hinchas o football hooligans. En los documentos no hay ninguna definición de hooliganism. Más bien, para Tsoukala ${ }^{66}$, la estigmatización de los hooligans está acompañada por un proceso definitorio fragmentado que atañe a la seguridad a nivel nacional e internacional ${ }^{67}$, así que hay confluencias significativas entre las políticas de contraste al hooliganism en los ámbitos europeo, nacional y local ${ }^{68}$. A partir de la desgracia del Heysel en 1985, las medidas

\footnotetext{
65. J. Apap, S. Carrera, "Maintaining Security within Borders: Towards a Permanent State of Emergency in the European Union?, en Alternatives. Global, Local, Political, 29, 2004, pp. 339-416.

66. A. Tsoukala, Football Hooliganism in Europe, Palgrave Macmillan, New York, 2009.

67. Ibid., p. 6.

68. Ya hablamos de esto en T. Pitch, Contro il decoro.
} 
de prevención situacional, basadas en la segregación y en la vigilancia de los espectadores de fútbol, han aumentado en términos temporales (antes y después de los partidos), espaciales (lugares externos al estadio) y de poblaciones afectadas. Han aumentado las penas y las medidas represivas de tipo administrativo para actos que no se considerarían delitos o amenazas fuera de un acontecimiento deportivo ${ }^{69}$ ). Por lo tanto, las recomendaciones del Consejo Europeo antes citadas no solo se aplican a perturbadores potenciales y a posibles manifestantes, sino también a los espectadores de fútbol y de otros acontecimientos deportivos; de hecho, el Consejo Europeo (2001) considera tanto al "football hooliganism" como a las manifestaciones políticas "amenazas a la seguridad urbana”.

No consideramos que la interacción entre los tres niveles (local, nacional y europeo) haya sido estudiada. Sin embargo, si los estudiamos de forma conjunta, estos niveles reflejan una ciudadanía limitada y a la que le han sido suprimidos algunos de aquellos derechos que, para Marshall, constituirían la misma ciudadanía moderna. En cambio, en esos tres niveles se destaca no solo una división entre "nosotros" y "ellos", sino también la construcción de comunidades basadas en el miedo y en la desconfianza hacia quien es diferente de "nosotros". Esta construcción choca tanto con las constituciones nacionales como con la Carta Europea de los derechos fundamentales, porque ellas subrayan, en cambio, una ciudadanía de tendencia inclusiva. En particular, incluso con sus límites, el Tratado de Niza parece seguir haciendo referencia al llamado modelo social europeo. Ese tratado no presupone una comunidad política ya existente, con valores y normas homogéneos que hay que defender de corrupciones “ajenas”; más bien, al menos por lo general, el mismo Tratado debería construir esta comunidad.

La relación entre políticas de seguridad e ideas de ciudadanía es muy estrecha y se necesita profundizarla. Tal y como dice Sassen ${ }^{70}$, la ciudadanía de las constituciones de la posguerra está en crisis, y los derechos de los que los ciudadanos "regulares" gozan se erosionan de manera constante. Mientras, para Sassen, las grandes corporations y la elite global no necesitan tener una ciudadanía jurídica para lograr lo que quieran, al mismo tiempo a la mayoría de los migrantes -a pesar de formar parte de la ciudadanía de algún país- se les trata como si no tuvieran derechos; incluso son expulsados de sus espacios vitales porque empresas estadounidenses, europeas y asiáticas requisan las tierras en sus países ${ }^{71}$. De ahí que la ciudadanía se deba reconsiderar en la base-claro está- de las políticas de seguridad.

69. A. Tsoukala, Football Hooliganism in Europe.

70. S. Sassen, “Who Are We, the Citizens?", en European Alternatives, 8 de octubre. 2014, disponible en http://euroalter. com.

71. Ibid., p. 1. 


\section{Lo que falta}

Ni el tema de la seguridad, ni tampoco las relaciones entre políticas de seguridad y ciudadanía pueden ser analizadas correctamente sin considerar el género, una perspectiva fundamental y que falta en la literatura sobre la materia. Esto es realmente paradójico, puesto que la mayoría de las justificaciones de las políticas de seguridad se refiere a la necesidad de proteger a "nuestras" mujeres. Sin embargo, una perspectiva que considere el género es también la única que puede estudiar por completo los efectos de esas políticas sobre el estatuto de ciudadanía de sujetos diferentes con respecto a la clase social, a la edad, al color de la piel, a la orientación sexual y a todas sus variables que definen la pluralidad de individuos que comparten hoy el mismo territorio, urbano, nacional y europeo.

El empleo del género femenino en las retóricas de la seguridad funciona muy bien para explicar la relación entre neoliberalismo y neoconservadurismo, y en este sentido analizar el caso italiano podría ser útil, con su faceta que llamaríamos neoliberal y la presencia de una noción de libertad que pretende ampliarse all the way down, en nombre del carácter sagrado de la elección, entendida como posibilidad de vender y comprar. Desde este punto de vista, la observación "Quién esté sentada sobre un tesoro, ¿por qué no debería emplearlo?”, de un famoso periodista del periódico Corriere della Sera es muy explicativa. La emancipación de las mujeres se considera como libertad de venderse. Lo femenino refleja la ideología dominante: se dice que en eso consiste la libertad, es decir, en la posibilidad de poner en práctica elecciones basadas en un cálculo de costes y beneficios, y si respecto de la mercantilización de las partes de su propio cuerpo los beneficios superan los costes, pues bien es justo perseguir esos beneficios. En este sentido, no solo todo puede convertirse en mercancía, sino -si puede- debe hacerlo. Al mismo tiempo -y solo parcialmente de forma contradictoria- lo femenino es la víctima por excelencia, ya sea por ser víctima potencial de los “malos", los que no son como "nosotros", los extranjeros, los migrantes, y como emblema y metáfora de la comunidad (ficticia) amenazada: la "cultura" nacional o local, la tradición, la lengua, la religión. La expresión "nuestras mujeres" significa todo esto. De ahí sale la faceta neoconservadora, que en Italia en los últimos años ha adquirido rasgos claramente racistas mediante el partido Liga Norte. Además, el reciente “descubrimiento" (en Italia) de la violencia masculina contra las mujeres, sobre todo la intrafamiliar o relativa a las relaciones íntimas, ha llevado a una importante campaña cuyo leitmotiv son la vulnerabilidad, la debilidad y la fragilidad femenina. Protección, tutela, seguridad para las 
"víctimas" o potenciales víctimas son las palabras clave que han desembocado en un decreto en el que normas para contrastar el llamado "feminicidio" andan con normas que militarizan, en nombre de la "seguridad", las obras del tren de alta velocidad del Valle de Susa (obra muy críticada y que el Gobierno quiere proteger incluso con el Ejército, si fuera necesario).

Así que las mujeres y lo femenino siempre se evocan en los discursos y respecto de los dispositivos de seguridad, a veces en sentido metafórico y a menudo muy concretamente, como en este último caso. Uno de los problemas es que hay mujeres y movimientos de las mujeres que entran en ese juego, o bien no se dan cuenta de eso. Ya ponía de manifiesto este problema en 1989, a propósito de la campaña contra el estupro. Sin embargo, ahora las desviaciones securitarias de un discurso basado en la victimización son mucho más evidentes que hace 35 años. Está claro que siempre se logra tener visibilidad, y esto puede interesar a alguien. Sin embargo, las consecuencias para la construcción de lo femenino y para la concreta libertad de las mujeres pueden ser y son muy profundas. En Italia, el discurso securitario saca provecho de las campañas sobre el "feminicidio" organizadas por las mujeres, y en Francia por las campañas contra la prostitución, también organizadas por mujeres. No hay duda de que la situación es muy compleja y delicada y, en ambos casos, puede ocurrir que el escenario político se reduzca al antagonismo entre buenos y malos, algo típico de los procesos de securitización y en total coherencia con la racionalidad política neoconservadora. De ahí que lo femenino se convierta en eje de la relación entre neoliberalismo y neoconservadurismo.

Además, hay una paradoja muy evidente en la idea de que la protección de las mujeres de la violencia masculina pueda ser confiada a las políticas securitarias, porque si quisiéramos proteger realmente a las mujeres con las medidas previstas por las políticas de seguridad, entonces deberíamos detener a todos los hombres, o bien ponernos a una policía hembra en casa ${ }^{72}$. Analizar esa paradoja es importante, puesto que más de la mitad de la población humana está compuesta por mujeres. En cambio, también en este caso el sujeto estándar cuya protección justifica las políticas de seguridad es un sujeto masculino, no reconocido como tal. Es verdad que las violencias contra las mujeres se emplean como pretexto para nuevas y mayores políticas represivas, pero la insistencia en la esterilización del espacio urbano, en la lucha contra las figuras típicas del "miedo" (mendigos, gitanos, extranjeros, drogadictos, etc.) atañe a un sujeto masculino, no demasiado joven, no demasiado pobre, que no necesita protegerse en su casa, sino solo

72. T. Pitch, C. Ventimiglia, Che genere di sicurezza. 
en los espacios públicos de desconocidos. Por el contrario, las mujeres tienen que tener mucho más cuidado con quien conocen bien, en su propia casa, más en los lugares de trabajo que en el espacio público (del cual no pueden gozar, sobre todo en algunas zonas y a unos horarios determinados) y con desconocidos. En realidad, la conciencia de que la violencia procede sobre todo de los hombres, en perjuicio tanto de las mujeres como de otros hombres, aún no ha entrado completamente en los proyectos de las políticas públicas, así como en los estudios científicos, críticos y no críticos, sobre la criminalidad, la seguridad, el riesgo, etc.

\section{Seguridad y el tema criminal}

Así que el tema de la "seguridad" protagoniza el estudio de los cambios de la racionalidad política hoy día dominante, tanto en el ámbito global como continental (Europa, Estados Unidos), estatal y local. Tal vez ahora podamos definir el tema de la seguridad tal y como hicimos con "el tema criminal"73, es decir, un ámbito de confines fluidos y siempre cambiantes, en el que se encuentran y chocan discursos, prácticas, políticas, saberes, pero también instituciones y actores colectivos, empresas privadas, movimientos sociales, ONG. Y, obviamente, los saberes, los discursos, las prácticas de seguridad, la acepción dominante de seguridad son analizados y (a veces) contestados no solo por estudiosos y políticos, sino también y sobre todo por actores colectivos, en el mismo ámbito en que las políticas de seguridad son implementadas. Esta contestación puede ser directa y consciente, pero también puede ser una resistencia difundida, no considerada como tal. Ya mencionamos ${ }^{74}$ que el conflicto relativo al "orden público” era un conflicto explícitamente político, que atañía -más o menos de manera consciente- a la concepción dominante del orden social. Pero cuando el orden público se esconde detrás de la seguridad de cada cual, lo que se esconde es con precisión la referencia a las posiciones de poder, lo cual dificulta la contestación. En este sentido hay despolitización en los procesos de securitización, tal y como se evidencia en los critical security studies. Sin embargo, conflictos y resistencia igual se dan.

El tema de la seguridad incide mucho en el criminal, reformula sus confines, porque en nombre de la seguridad (de la emergencia, entre otros) no solo se criminalizan nuevos comportamientos, sino sobre todo se toman medidas administrativas que se aplican 
a comportamientos no considerados crímenes y en la base de los cuales muchos grupos de personas se clasifican como potencialmente peligrosos, para vigilar, para alejar de los "buenos"75. El paradigma securitario se basa en el principio de prevención, pero no por medio de la amenaza de la pena, sino a través de un complejo conjunto de dispositivos diferentes, algunos públicos, otros privados, que no solo aspiran a frenar a los delincuentes potenciales, sino más bien a defender a los que no lo son. El mismo ámbito penal cambia de sentido y naturaleza, adquiriendo la doble cara de simple neutralización de los condenados y de indemnización moral de las víctimas. En realidad, cada vez más a menudo tanto lo penal como las medidas securitarias tienen el objetivo de "tranquilizar" y crear consenso mediante la simple añadidura tanto de las normas como de las medidas de seguridad, lo cual no significa que sus efectos reales no puedan ser incluso muy fuertes. En el centro de todos estos dispositivos y de las retóricas que los justifican está la "víctima", real y potencial, es decir, todos los hombres "buenos". La importancia actual del "peligro" (y no solo del riesgo) parece recordar los paradigmas de la defensa social e incluso de la escuela positiva, pero ahora el peligro está por doquier y no bastan las paredes de cárceles y manicomios para contenerlo; tal vez la construcción de muros, reales y simbólicos, se necesite para defendernos a nosotros mismos, para alejar físicamente el "peligro". A su vez, el peligro no necesita ser definido de manera precisa o que esté relacionado con comportamientos verificables empíricamente, lo cual lo convierte en más preocupante y lo pone en relación cada vez con figuras y fenómenos diferentes. Alejar el peligro no significa necesariamente analizar sus eventuales causas y buscar su solución. Es como si dijéramos: alejemos de las ciudades a los gitanos y a los sinti, poniéndolos en guetos y que se las arreglen ellos...

Traducción del italiano de M. Colucciello

75. Los criminólogos se han interesado mucho por este tema; véase sobre todo cfr. D. Garland, The Culture of Control. 